\title{
Características sociodemográficas e histopatológicas en pacientes con cáncer gástrico en una población con alto riesgo en Colombia
}

\section{Sociodemographic and histopathological characteristics of patients with gastric cancer in a high-risk population in Colombia}

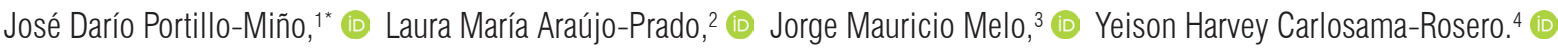

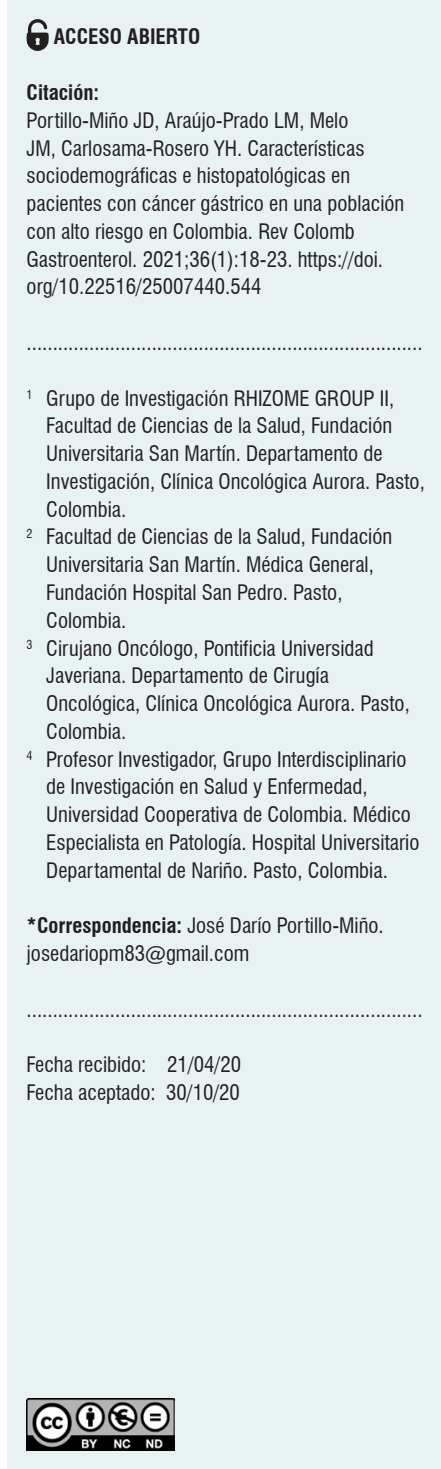

\section{Resumen}

Objetivo: describir las características sociodemográficas e histopatológicas en pacientes con diagnóstico de adenocarcinoma gástrico en la Clínica Oncológica Aurora, durante el período 2014-2017 en la ciudad de Pasto, Colombia. Métodos: se realizó un estudio descriptivo en una cohorte de 54 pacientes con diagnóstico de cáncer gástrico sometidos a gastrectomía durante los años 2014 a 2017. La información sociodemográfica se obtuvo a través de la historia clínica. El sistema de Sydney y la clasificación de Lauren se usaron para determinar las características histopatológicas. Resultados: la mayoría de los tumores se presentó en hombres mayores de 50 años (relación hombre mujer de 2,6:1). La ubicación predominante fue la región antropilórica. El histotipo tumoral más frecuente fue el intestinal ( $80 \%$ ). La prevalencia de Helicobacter pylori en pacientes fue del $24,07 \%$ y fue mayor en el histotipo intestinal. La metaplasia intestinal fue la lesión premaligna más prevalente en todos los histotipos tumorales. Conclusiones: en el presente estudio se encontró que la edad mayor a 50 años y el sexo masculino son condiciones asociadas con el cáncer gástrico; hallazgo ya demostrado en estudios previos. Es perentorio avanzar en el mejoramiento de las condiciones de salud pública, control de la infección por $\mathrm{H}$. pylori y tamizaje temprano de lesiones premalignas, pues son factores determinantes en la carcinogénesis de pacientes con carcinomas no cardiales e histotipos intestinales ubicados en la región antrocorporal. Aunque no se encontraron diferencias significativas entre los histotipos tumorales, el adenocarcinoma de tipo intestinal ubicado en la región antropilórica fue el diagnóstico más frecuente.

\section{Palabras clave \\ Helicobacter pylori, gastrectomía, cáncer gástrico, histopatología.}

\begin{abstract}
Objective: To describe the sociodemographic and histopathological characteristics of patients diagnosed with gastric adenocarcinoma at the Clínica Oncológica Aurora between 2014 and 2017 in the city of Pasto, Colombia. Methodology: A descriptive study was carried out in a cohort of 54 patients diagnosed with gastric cancer who underwent gastrectomy between 2014 and 2017. Sociodemographic information was taken from the medical records of the patients. The Sydney system and Lauren classification were used to determine histopathological characteristics. Results: Most tumors were found in men older than 50 years (with a male:female ratio of 2.6:1). The predominant location was the antropyloric region. The most frequent tumor histotype was intestinal $(80 \%)$. The prevalence of Helicobacter pylori in patients was $24.07 \%$ and it was most commonly found in the intestinal histotype. Intestinal metaplasia was the most prevalent premalignant lesion in all tumor histotypes. Conclusions: The present study found that the age of 50 and the male sex are conditions associated with gastric cancer. This finding has already been demonstrated in previous studies. Progress in improving public health conditions, controlling $\mathrm{H}$. pylori infection, and early screening of premalignant lesions is imperative, as they are determining factors of carcinogenesis in patients with non-cardia carcinomas and intestinal histotypes located in the anthrocorporal region. Although no significant differences were found among tumor histotypes, intestinal adenocarcinoma in the antropyloric region was the most frequent diagnosis.
\end{abstract}

\section{Keywords}

Helicobacter pylori, Gastrectomy, Gastric cancer, Histopathology. 


\section{INTRODUCCIÓN}

El cáncer gástrico representa el quinto cáncer diagnosticado más frecuente y la tercera causa de muerte atribuible al cáncer en todo el mundo (1). Las áreas de alto riesgo para desarrollar cáncer gástrico son el Este de Asia (China, Corea, Mongolia y Japón) (1), Europa Oriental, América Central y Sudamérica (2).

De acuerdo con la información derivada de los registros poblacionales de cáncer en Colombia, el cáncer gástrico es una patología de alto impacto en la población y se destaca la afectación en el suroccidente colombiano, especialmente en el sexo masculino. Por ejemplo, se ha visto una tasa de incidencia de cáncer gástrico en hombres en la ciudad de Pasto de 26,7/100 000 habitantes cada año (3). Además, se han descrito otros factores de riesgo como el bajo nivel educativo, la pobreza, la elevada ingesta de sal, el bajo consumo de antioxidantes, la presencia de lesiones histológicas premalignas como la atrofia y la metaplasia intestinal, y la inexorable infección por Helicobacter pylori $(4,5)$. No obstante, la asociación con los distintos factores de riesgo es dependiente de los histotipos tumorales.

Según la clasificación de Lauren y Jarvi, los adenocarcinomas se clasifican en intestinal y difuso (6); esta clasificación es muy relevante debido a que son tumores de origen epitelial, pero tienen algunas diferencias. Por ende, el carcinoma tipo intestinal es más común en hombres de raza negra y en grupos de personas de edad avanzada; además está asociado con factores medioambientales, deficientes condiciones de salud pública y lesiones premalignas e infección por $H$. pylori. Este tipo de cáncer también evidencia grandes variaciones geográficas y es más frecuente en la región antropilórica (7). Por su parte, el histotipo difuso no guarda relación con condiciones medioambientales, lesiones premalignas o infección por $H$. pylori; se ha mencionado en este tipo tumoral una mayor incidencia en mujeres y una habitual asociación con la mutación de la E-cadherina (8).

En el suroccidente colombiano se han realizado algunas investigaciones donde se exploran las características sociodemográficas y clínicas de pacientes con cáncer gástrico o lesiones premalignas $(9,10)$; sin embargo, según la revisión de la literatura realizada por los autores, no se encontraron publicaciones de las características histopatológicas y sociodemográficas en pacientes con cáncer gástrico en el departamento de Nariño. En ese orden de ideas, se planteó un estudio descriptivo en el que se analizan las características sociodemográficas y microscópicas de pacientes con cáncer gástrico y se indaga sobre la presunta relación de estas variables con el histotipo tumoral.

\section{MATERIALES Y MÉTODOS}

Se llevó a cabo un estudio descriptivo con la concerniente recolección de la información sociodemográfica e histopatológica de forma retrospectiva. Se incluyó la totalidad de los pacientes diagnosticados con cáncer gástrico y sometidos a gastrectomía total y subtotal en la Clínica Oncológica Aurora, en Pasto, en los años 2014-2017. La información sociodemográfica se obtuvo de la historia clínica, mientras que la información histopatológica se obtuvo de los reportes de patología. En las variables sociodemográficas se incluyeron edad, sexo, etnia, estado civil, procedencia, régimen de afiliación al sistema de salud y nivel educativo. El diagnóstico histopatológico se realizó en cortes incluidos en parafina y teñidos posteriormente con la coloración de hematoxilina-eosina. Se determinaron variables como ubicación del tumor, presencia de infección por H. pylori, presencia de lesiones premalignas asociadas y determinación del histotipo tumoral. La clasificación de las lesiones premalignas se realizó de acuerdo con el sistema de Sydney, mientras que la categorización de los histotipos se efectuó mediante el sistema de Lauren clasificando los tumores como intestinales o difusos. Cuando en el estudio microscópico se demostraron los dos componentes (intestinal y difuso en una extensión del 50 \% cada uno), se clasificó como un tumor mixto.

Para la variable edad se calculó la media y la desviación estándar. En las variables categóricas se determinaron las frecuencias absolutas y las proporciones. Las diferencias entre las medias de edad en los distintos histotipos tumorales se realizó mediante la prueba de Anova de un factor. Se compararon las proporciones de las variables de interés entre los histotipos tumorales mediante la prueba de chi cuadrado $\left(\chi^{2}\right)$. Se consideraron como significativos valores de $p<0,05$. Se empleó el programa SPSS versión 25 para los análisis estadísticos.

\section{RESULTADOS}

En el período de estudio se recolectó la información de 54 pacientes con edades entre los 26 y 88 años. El promedio de edad fue de 64 años con una desviación estándar de 13,2 años. La mayoría de los tumores ocurrió en pacientes del sexo masculino, con una relación hombre mujer de 2,6:1. Las demás características sociodemográficas se muestran en la Tabla 1.

La paciente más joven correspondía a una mujer de 26 años con un adenocarcinoma de tipo difuso, mientras que el paciente de mayor edad corresponde a un hombre de 88 años con un adenocarcinoma de tipo intestinal. 
Tabla 1. Características sociodemográficas de la población de estudio

\begin{tabular}{|c|c|c|c|}
\hline \multicolumn{2}{|c|}{ Variable } & $\mathbf{n}$ & $\%$ \\
\hline \multirow[t]{2}{*}{ Sexo } & Femenino & 15 & 27,3 \\
\hline & Masculino & 39 & 70,9 \\
\hline \multirow[t]{4}{*}{ Etnia } & Negro & 3 & 5,5 \\
\hline & Indígena & 6 & 10,9 \\
\hline & Romaní & 1 & 1,8 \\
\hline & Mestizo & 44 & 80 \\
\hline \multirow[t]{6}{*}{ Estado civil } & Soltero & 2 & 3,6 \\
\hline & Casado & 17 & 30,9 \\
\hline & Unión libre & 5 & 9,1 \\
\hline & Divorciado & 5 & 9,1 \\
\hline & Viudo & 13 & 23,6 \\
\hline & Sin especificar & 12 & 21,8 \\
\hline \multirow[t]{2}{*}{ Procedencia } & Urbano & 36 & 66,7 \\
\hline & Rural & 18 & 33,3 \\
\hline \multirow[t]{2}{*}{ Régimen } & Contributivo & 28 & 50,9 \\
\hline & Subsidiado & 26 & 47,3 \\
\hline \multirow[t]{5}{*}{ Nivel educativo } & Universitario & 2 & 3,6 \\
\hline & Secundaria & 20 & 36,4 \\
\hline & Primaria & 16 & 29,5 \\
\hline & Ninguno & 14 & 25,5 \\
\hline & Sin especificar & 2 & 3,6 \\
\hline
\end{tabular}

La clasificación microscópica de los tumores gástricos se muestra en la Figura 1.

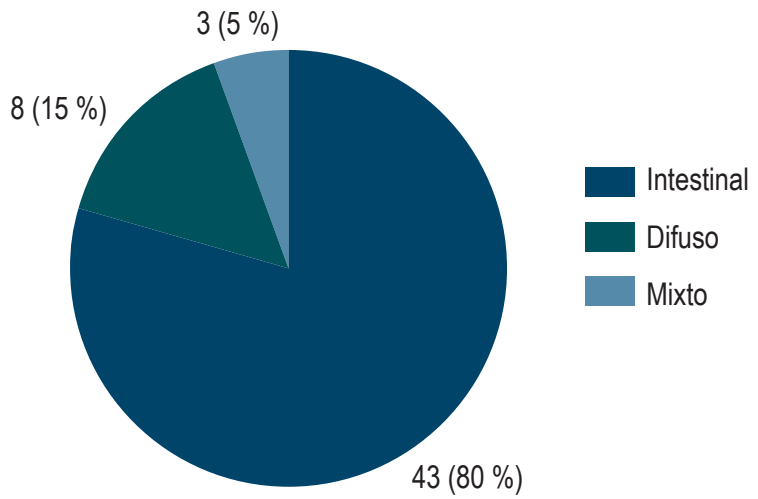

Figura 1. Distribución de los tumores de acuerdo con el histotipo según la clasificación de Lauren.

La media de la edad para el histotipo intestinal fue de 63 años, para el histotipo difuso fue de 64 y para el histotipo mixto fue de 73 años. La prueba de Anova de un factor no demostró diferencias significativas entre las medias de edad $(p=0,492)$.

Finalmente, se exploró la relación de variables como la edad categorizada ( $<50$ años $y \geq 50$ años), el sexo y las variables histopatológicas con los distintos histotipos tumorales sin evidenciarse diferencias estadísticamente significativas
(Tabla 2). Se documentó la presencia de H. pylori en 13 casos, que corresponden al $24,07 \%$ de los pacientes.

Tabla 2. Relación de la edad, el sexo y las características anatomopatológicas con los histotipos tumorales

\begin{tabular}{llcccc}
\hline & Variable & Intestinal & Difuso & Mixto & $\begin{array}{c}\text { Valor } \\
\text { de } \boldsymbol{p}^{*}\end{array}$ \\
\hline Edad & $<50$ años & $6(14)$ & $0(0)$ & $0(0)$ & 0,422 \\
& $\geq 50$ años & $37(86)$ & $8(100)$ & $3(100)$ & \\
Sexo & Femenino & $13(30,2)$ & $2(25)$ & $0(0)$ & 0,518 \\
& Masculino & $30(69,8)$ & $6(75)$ & $3(100)$ & \\
Ubicación & Cardias & $2(4,7)$ & $0(0)$ & $0(0)$ & 0,390 \\
del tumor & Cuerpo y fondo & $16(37,2)$ & $1(12,5)$ & $0(0)$ & \\
& Antro & $11(25,6)$ & $2(25)$ & $1(33,3)$ & \\
& Antro y piloro & $10(23,3)$ & $5(62,5)$ & $2(66,7)$ & \\
& No especificado & $4(9,3)$ & $0(0)$ & $0(0)$ & \\
H. pylori & Ausente & $31(72,1)$ & $7(87,5)$ & $3(100)$ & 0,390 \\
& Presente & $12(27,9)$ & $1(12,5)$ & $0(0)$ & \\
& & $1(2,3)$ & $0(0)$ & $0(0)$ & 0,970 \\
Lesión & Atrofia & $41(95,4)$ & $8(100)$ & $3(100)$ & \\
premaligna & Metaplasia & $1(2,3)$ & $0(0)$ & $0(0)$ & \\
asociada & Ninguna & & &
\end{tabular}

*Un valor de $p<0,05$ se consideró estadísticamente significativo.

\section{DISCUSIÓN}

El cáncer gástrico es una enfermedad multifactorial en la que se han descrito componentes del huésped y medioambientales, y la infección por $H$. pylori (11) como contribuyentes de la patología. Entre los factores del hospedero, se ha detallado la edad > 50 años y el sexo masculino como variables asociadas con el cáncer gástrico (12), características que se encuentran en consonancia con los resultados reportados en este trabajo. La prevalencia del cáncer de acuerdo con el sexo es variable en países desarrollados donde la relación es de 2,2, o en países en desarrollo donde la proporción es de 1,8 (13). En el presente estudio se demostró una relación hombre-mujer de 2,6, en la que se destaca el sexo masculino como un importante factor de riesgo para el desarrollo de la patología. No obstante, algunos autores han descrito que el adenocarcinoma de tipo difuso es más frecuente en pacientes jóvenes del sexo femenino $(14,15)$; dicha relación no se definió en la presente investigación, en la que la totalidad de los histotipos difusos ( 8 casos) se presentaron en pacientes mayores de 50 años y el $75 \%$ de los mismos (6 casos) ocurrieron en pacientes del sexo masculino. De manera similar, la totalidad de los casos pertenecientes al histotipo mixto corresponden a pacientes del sexo masculino mayores de 50 años. Estos hallazgos resaltan la importancia del sexo y la edad en la carcinogénesis gástrica. 
La relación de la edad con el desarrollo de cáncer está asociada con una mayor inestabilidad genómica producto de condiciones como el envejecimiento celular, la respuesta inflamatoria crónica en la mucosa gástrica, las modificaciones del microambiente tisular producto de la dieta y los cambios directos e indirectos inducidos por $H$. pylori. Dicha inestabilidad genómica produciría dos mecanismos moleculares: la inestabilidad de microsatélites y la inestabilidad intracromosómica; la primera se encuentra relacionada con la incapacidad para reparar el ácido desoxirribonucleico $(\mathrm{ADN})$ dañado, mientras que la segunda se relaciona con alteraciones en oncogenes y genes supresores de tumores $(16,17)$. Se han citado algunas alteraciones genéticas relacionadas con el histotipo tumoral; en ese sentido, son más comunes las alteraciones de la E- cadherina en el carcinoma de tipo difuso, mientras que las mutaciones del gen Erb se asocian con más frecuencia al adenocarcinoma gástrico de tipo intestinal $(7,8)$. Estos hallazgos son trascendentales porque sugieren dos vías de progresión tumoral diferentes. En nuestro estudio destacamos la presencia de un adenocarcinoma de tipo difuso en la paciente más joven, correspondiente a una mujer de 26 años; la presencia de este tipo de cáncer en un paciente joven debe fomentar la sospecha clínica debido a la asociación que existe con mutaciones en la línea germinal de la E-cadherina y cadherina-1 (CDH1) con el síndrome de cáncer gástrico hereditario difuso (18). Por su parte, los tumores que aparecen después de los 50 años ocurren probablemente como resultado de mutaciones e inestabilidad genómica acumulativa a lo largo de los años en la línea somática.

En nuestro trabajo es interesante la relativa baja prevalencia de infección por $H$. pylori $(24 \%)$ demostrada en el estudio histopatológico. Este hallazgo contrasta con los datos reportados por Hooi y colaboradores en un reciente metaanálisis en el que se estiman prevalencias de infección por H. pylori para África del 79,1\%; Asia, 54,7 \%; y Latinoamérica y el Caribe, 63,4 \% (19). En Colombia, posiblemente, el estudio más relevante en esta materia es el realizado por Bravo LE y colaboradores (20), según el cual la prevalencia en general de $H$. pylori fue del $69,1 \%$ y la prevalencia de la infección en pacientes con cáncer fue del 40,2 \% (21). Como posibles explicaciones a la baja prevalencia de $H$. pylori en este estudio, los autores consideran los siguientes factores: el primero, relacionado con el tratamiento antimicrobiano previo a la cirugía; el segundo, la presencia de metaplasia intestinal en casi la totalidad de los pacientes y la evaluación de histotipos como el difuso o mixto que clásicamente no guardan correlación con la infección bacteriana. Infortunadamente, no fue posible documentar la instauración de tratamientos prequirúrgicos anti-H. pylori, dado que la clínica es un centro de referencia en cirugía oncológica y no se dispone de la historia clínica completa de los pacientes atendidos en otras instituciones donde presuntamente recibieron tratamiento farmacológico. Del mismo modo, es bien conocida la dificultad de documentar H. pylori en mucosas gástricas con metaplasia intestinal (21), puesto que este hecho estaría condicionado porque el epitelio intestinal metaplásico, al parecer, resulta en un medio ambiente hostil para la bacteria al limitar su capacidad para sintetizar amonio; además, se ha sugerido que, debido a la inflamación crónica y el daño tisular provocado por la bacteria, si estuviera presente en esta faceta de la carcinogénesis, es muy difícil su identificación; y finalmente, la infección por $H$. pylori se asocia con el histotipo intestinal y no con el histotipo difuso $(22,23)$.

Como se pudo establecer en esta investigación, la infección se documentó en el $28 \%$ de los pacientes con el histotipo intestinal y tan solo en el $12 \%$ de los pacientes con histotipo difuso. En contraposición a lo descrito en países desarrollados donde se ha demostrado una prevalencia creciente de carcinomas en ubicaciones como la cardial y corpofúndica $(24,25)$, en este estudio encontramos que la localización antropilórica es el sitio más frecuente de ubicación del tumor; estos resultados concuerdan con los reportados por Adrada y colaboradores en el departamento del Cauca y han permanecido inalterados durante muchas décadas en el suroccidente colombiano (9). En ese sentido, varios autores han planteado dos tipos de carcinomas relacionados con la ubicación; es decir, los carcinomas cardiales más frecuentes en países desarrollados, con mejores condiciones de salud pública y asociados con dietas de tipo occidental, consumo de alcohol y obesidad; y carcinomas no cardiales, propios de países en vías de desarrollo y asociados con deficientes condiciones de salud pública, lesiones premalignas e infección por H. pylori $(13,26)$.

Entre las limitaciones del estudio, se debe tener en cuenta que se trató de una corte de pacientes pequeña; $\sin$ embargo, los hallazgos son consistentes con el comportamiento sociodemográfico e histopatológico de estudios de otras latitudes y con tamaños de muestra más amplios. De igual forma, no fue posible establecer si se realizó tratamiento contra $H$. pylori en los pacientes sometidos a gastrectomía; en ese orden de ideas, es necesario realizar futuras investigaciones que permitan determinar si la erradicación del microorganismo se llevó a cabo por el tratamiento previo, razón por la cual serían pertinentes estudios en biopsias y pruebas de aliento previos para verificar la infección por H. pylori. 


\section{CONCLUSIONES}

Nuestros hallazgos plantean que se debe avanzar en el mejoramiento de las condiciones de salud pública, control de la infección por $H$. pylori y tamizaje temprano de lesiones premalignas, puesto que son factores determinantes en la carcinogénesis en los pacientes con carcinomas no cardiales e histotipos intestinales ubicados en la región antrocorporal. La edad mayor de 50 años y el sexo masculino son condiciones asociadas con el cáncer gástrico en la población objeto de estudio. Aunque no se demostraron diferencias significativas entre los histotipos tumorales, el adenocarcinoma de tipo intestinal ubicado en la región antropilórica fue el diagnóstico más frecuentemente.

\section{Agradecimientos}

A la Clínica Oncológica Aurora, en Pasto, Colombia, por permitirnos desarrollar esta investigación; y a los pacientes, por sus invaluables aportes.

\section{Conflicto de intereses}

Los autores declaran no tener ningún conflicto de interés.

\section{Financiación}

El trabajo fue financiado con recursos propios de los autores.

\section{REFERENCIAS}

1. Bray F, Ferlay J, Soerjomataram I, Siegel RL, Torre LA, Jemal A. Global cancer statistics 2018: GLOBOCAN estimates of incidence and mortality worldwide for 36 cancers in 185 countries. CA Cancer J Clin. 2018;68(6):394-424. https://doi.org/10.3322/caac.21492

2. Parkin DM, Bray F, Ferlay J, Pisani P. Global cancer statistics, 2002. CA Cancer J Clin. 2005;55(2):74-108. https:// doi.org/10.3322/canjclin.55.2.74

3. Bravo LE, Muñoz N. Epidemiology of cancer in Colombia. Colomb Med (Cali). 2018;49(1):9-12. https: / / doi. org/10.25100/cm.v49i1.3877

4. Guggenheim DE, Shah MA. Gastric cancer epidemiology and risk factors. J Surg Oncol. 2013;107(3):230-6. https:// doi.org/10.1002/jso.23262

5. Kelley JR, Duggan JM. Gastric cancer epidemiology and risk factors. J Clin Epidemiol. 2003;56(1):1-9. https:// doi. org/10.1016/s0895-4356(02)00534-6

6. Lauren P. The two histological main types of gastric carcinoma: diffuse and so-called intestinal-type carcinoma. An attempt at a histo-clinical classification. Acta Pathol Microbiol Scand. 1965;64:31-49. https://doi. org/10.1111/apm.1965.64.1.31

7. Crew KD, Neugut AI. Epidemiology of gastric cancer. World J Gastroenterol. 2006;12(3):354-62. https://doi. org/10.3748/wjg.v12.i3.354

8. Correa P, Camargo MC, Piazuelo MB. Overview and Pathology of Gastric Cancer. En: Wang TC, Fox JG, Giraud AS (editores). The Biology of Gastric Cancers. Nueva York: Springer; 2009. p. 1-24. https: / doi.org/10.1007/978-0387-69182-4 1

9. Adrada JC, Calambás FH, Díaz JE, Delgado DO, Sierra CH. Características sociodemográficas y clínicas en una población con cáncer gástrico en el Cauca, Colombia. Rev Col Gastroenterol. 2008;23(4):309-314.
10. Bedoya U Á, Sansón G. F, Yépez Fuertes V. Y, Santacruz M. C, Cifuentes C. Y, Calvache P. D, et al. Prevalence and severity of gastric cancer precursor lesions in a high risk area. Rev Col Gastroenterol. 2012;27(4):275-281.

11. Zabaleta J. Multifactorial Etiology of Gastric Cancer. En: Dumitrescu RG, Verma M (editores). Cancer Epigenetics: Methods and Protocols. Totowa, NJ: Humana Press; 2012. p. 411-35. https://doi.org/10.1007/978-1-61779-612826

12. Yü J, He Y, Guo Z. Age trend of the male to female sex ratio in surgical gastric cancer patients at a single institution. World J Surg Oncol. 2014;12:269. https://doi. org/10.1186/1477-7819-12-269

13. Rawla P, Barsouk A. Epidemiology of gastric cancer: global trends, risk factors and prevention. Prz Gastroenterol. 2019;14(1):26-38. https://doi.org/10.5114/ pg.2018.80001

14. Zheng H, Takahashi H, Murai Y, Cui Z, Nomoto K, Miwa S, Tsuneyama K, Takano Y. Pathobiological characteristics of intestinal and diffuse-type gastric carcinoma in Japan: an immunostaining study on the tissue microarray. J Clin Pathol. 2007;60(3):273-7. https://doi.org/10.1136/ jcp.2006.038778

15. Rampazzo A, Mott GL, Fontana K, Fagundes RB. Gastric adenocarcinoma trends in the central region of Rio Grande do Sul (Southern Brazil): what has changed in 25 years? Arq Gastroenterol. 2012;49(3):178-83. https://doi. org/10.1590/s0004-28032012000300002

16. Halling KC, Harper J, Moskaluk CA, Thibodeau SN, Petroni GR, Yustein AS, Tosi P, Minacci C, Roviello F, Piva P, Hamilton SR, Jackson CE, Powell SM. Origin of microsatellite instability in gastric cancer. Am J Pathol. 1999; 155(1):205-11. https://doi.org/10.1016/S00029440(10)65114-0 
17. Nardone G. Review article: molecular basis of gastric carcinogenesis. Aliment Pharmacol Ther. 2003;17 Suppl 2:7581. https://doi.org/10.1046/j.1365-2036.17.s2.10.x

18. Bresciani C, Perez RO, Gama-Rodrigues J. Familial gastric cancer. Arq Gastroenterol. 2003;40(2):114-7. https://doi. org/10.1590/s0004-28032003000200010

19. Hooi JKY, Lai WY, Ng WK, Suen MMY, Underwood FE, Tanyingoh D, Malfertheiner P, Graham DY, Wong VWS, Wu JCY, Chan FKL, Sung JJY, Kaplan GG, Ng SC. Global Prevalence of Helicobacter pylori Infection: Systematic Review and Meta-Analysis. Gastroenterology. 2017;153(2):420-429. https://doi.org/10.1053/j.gastro.2017.04.022

20. Bravo LE, Cortés A, Carrascal E, Jaramillo R, García LS, Bravo PE, Badel A, Bravo PA. Helicobacter pylori: patología y prevalencia en biopsias gástricas en Colombia. Colombia Médica. 2003;34(3):124-131.

21. Chacaltana Mendoza A, Soriano Álvarez C, Frisancho Velarde O. Factores de riesgo asociados a metaplasia intestinal gástrica en pacientes sin enfermedad gastroduodenal significativa: ¿Está siempre asociada la infección por Helicobacter pylori? Rev Gastroenterol Perú. 2012;32(1):50-7.

22. van der Woude CJ, Kleibeuker JH, Tiebosch AT, Homan M, Beuving A, Jansen PL, Moshage H. Diffuse and intes- tinal type gastric carcinomas differ in their expression of apoptosis related proteins. J Clin Pathol. 2003;56(9):699702. https://doi.org/10.1136/jcp.56.9.699

23. Sipponen P, Kekki M, Siurala M. Atrophic chronic gastritis and intestinal metaplasia in gastric carcinoma. Comparison with a representative population sample. Cancer. 1983;52(6):1062-8. https://doi. org/10.1002/1097-0142(19830915)52:6<1062::aidcncr2820520622>3.0.co;2-p

24. Crew KD, Neugut AI. Epidemiology of gastric cancer. World J Gastroenterol. 2006;12(3):354-62. https://doi. org/10.3748/wjg.v12.i3.354

25. Sitarz R, Skierucha M, Mielko J, Offerhaus GJA, Maciejewski R, Polkowski WP. Gastric cancer: epidemiology, prevention, classification, and treatment. Cancer Manag Res. 2018;10:239-248. https://doi.org/10.2147/ CMAR.S149619

26. Corvalán A, Akiba S, Valenzuela MT, Cumsille MA, Koriyama C, Argandoña J, Backhouse C, Bal M, Mena F, Palma M, Eizuru Y. Características clínico-moleculares del cáncer gástrico cardial asociado al virus Epstein Barr. Rev Med Chil. 2005; 133(7):753-60. https://doi.org/10.4067/ s0034-98872005000700001 